See discussions, stats, and author profiles for this publication at: https://www.researchgate.net/publication/332078533

\title{
Modeling of a Cable-Based Revolute Joint Using Biphasic Media Variable Stiffness Actuation
}

Conference Paper · February 2019

DOI: $10.1109 /$ IRC.2019.00125

CITATIONS

0

5 authors, including:

Jesus Hiram Lugo Calles

Università degli Studi di Genova

8 PUBLICATIONS 7 CITATIONS

SEE PROFILE

Matteo Zoppi

Università degli Studi di Genova

169 PUBLICATIONS 949 CITATIONS

SEE PROFILE

Some of the authors of this publication are also working on these related projects:

Project tetrad-screw propelled omnidirectional all-terrain mobile robot View project

Project Screw-propelled locomotion View project
READS

69

Vishal Ramadoss

Università degli Studi di Genova

9 PUBLICATIONS 1 CITATION

SEE PROFILE

Rezia M. Molfino

Università degli Studi di Genova

285 PUBLICATIONS 1,206 CITATIONS

SEE PROFILE 


\title{
Modeling of a cable-based revolute joint using biphasic media variable stiffness actuation
}

\author{
Jesus H. Lugo*, Vishal Ramadoss, Matteo Zoppi, Giorgio Cannata and Rezia Molfino \\ PMAR Robotics \\ University of Genoa \\ Genoa, Italy- 16145 \\ *E-mail: lugocalles@dimec.unige.it
}

\begin{abstract}
In recent times, safe interactions between humans and robots are required for innumerable tasks and environments. This safety can be achieved using compliance design and control of mechanisms. Cable-driven mechanisms are used when applications need to have light structures, meaning that their actuators must be relocated to ground and forces are transferred along tensioned cables. This paper presents a compliant cable-driven revolute joint using biphasic media variable stiffness actuators. Actuator's stiffness is controlled by changing pressure of control fluid into distribution lines. The used control fluid is biphasic, composed of separated gas and liquid fractions with predefined ratio. The mathematical model of the actuator is presented along with its position and stiffness model-based control, then, equations relating to the dynamics of the mechanism are provided with a joint stiffness and orientation controller. Results from simulations are discussed.
\end{abstract}

\section{INTRODUCTION}

Stiffness control in actuators is needed when a robot must perform tasks in the presence of humans, or when collisions with the environment are possible. For these scenarios, velocity and position should be sufficiently accurate while minimizing the risk of damaging humans, environment and itself [1]. Applications that require controllable stiffness can be identified as "robot-human interaction" and "natural dynamics adjustment" [2]. The first one is focused on having a safer and more natural interaction between the human and the machine, while the second one deals with the adjustment of natural dynamics of the mechanical system in order to have a desired natural motion to reduce the energy consumption.

Cable-driven manipulators are characterized by lightweight structure and adjustable stiffness. In [3], a compact variable stiffness device (VSD) is proposed to optimally control the stiffness of a two DoF cable-driven joint. VSD units with nonlinear stiffness characteristics have been developed to adjust the compliance of cable-driven manipulators in [4], [5]. The total stiffness is given from cable stiffness and VSD by attaching in series a VSD along each cable of the manipulator. Robot's stiffness can thus be controlled by manipulating cable tensions. A cable-based active variable stiffness module is presented in [6]; its main characteristic is to have independent stiffness modulation with the desired tension due to controlled changes in structural parameters. A comparison of modulartype antagonistic tendon-driven joints (TDJ) is detailed in [7] in order to design a new joint with higher stiffness and larger travel range than conventional TDJs.

Biphasic media variable stiffness actuators (BMVSA) have been extensively studied in $[\overline{8}]-[15]$. These devices were designed using pneumatic and hydraulic components. A control fluid, composed of two different non-mixable fluids, must be used in order to transmit motion into the circuit. The schematic diagram shown in Fig. 1 belongs to the biphasic fluid model, having liquid and gas fractions. The gas works as nonlinear elastic element, altering the stiffness due to pressure gas variations, while the liquid one is assumed incompressible and used to provide pressure changes and motion to the output link. The control fluid encompasses two different fluids that can be both compressible; in this case, mineral oil and Nitrogen, having the second one secluded inside the accumulators. As mentioned in [11], it is preferable that the gas is close to surface of the output link of the actuator, since the volume of liquid involved when an external force moves the output link and changes the pressures of the gas fractions is minimum. Figure 1 shows a design that is convenient due to its modularity, where the gas fraction is contained in hydropneumatic accumulators (HPAs) that can be easily replaced according to the requiered range of compliance. Some implementations of the system are industrial grippers for garment handling [12][14], surgical robotics concepts, suspension systems for mobile platforms [11] and actuation of revolute joints [10]. Pressure and flow control valves are included in the hydraulic circuit with aim of controlling stiffness of the actuator. BMVSA can be considered to have antagonistic controlled stiffness, where two actuators with non-adaptable stiffness and nonlinear forcedisplacement characteristics are coupled antagonistically. Finally, it is assumed that the gas is ideal and the process is isothermal. The paper is organized as follows: Section II depicts the mathematical modeling of BMVSA and proposes a model-based controller for position and stiffness. Section III shows geometric and dynamic characteristics of the revolute joint. Section IV discusses the results from simulations of the system. Section V provides conclusions and ongoing work.

\section{Modeling OF BMVSA}

The BMVSA equation of motion is described in 11, where $m$ is the mass of the output link of the actuator, (piston, seals and mechanical attachments), $x_{p}$ is the displacement of the piston, $k_{v}$ is the coefficient of viscosity, $F_{p}$ is the force 


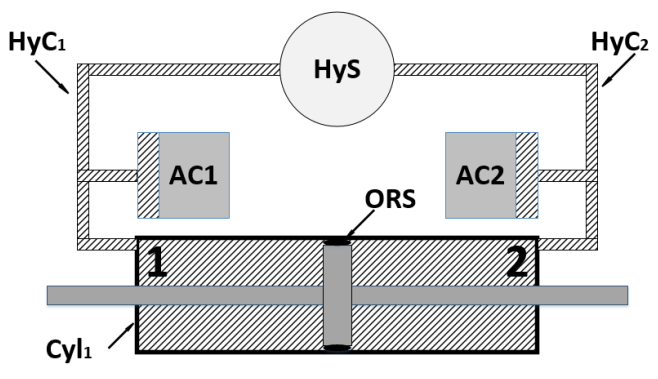

Fig. 1: BMVSA diagram. The actuator is composed of a double acting hydraulic cylinder $(\mathrm{Cyll})$ connected to a hydraulic circuit. This circuit includes a supply and distribution system $(\mathrm{HyS})$ and two hydraulic distribution lines $(\mathrm{HyCl}$ and $\mathrm{HyC2}$ ) respectively connected to the cylinder's chambers and the insulated HPAs ( $A C l$ and $A C 2$ ). O-ring type seals (ORS) provide the translation of the piston without leakage between the chambers.

generated by the difference of pressures $P_{1}$ and $P_{2}$, and $F$ is an external force. The subscripts 1 and 2 denote chambers 1 and 2 and their corresponding HPAs.

$$
m \ddot{x}_{p}+k_{v} \dot{x}_{p}-F_{p}=F
$$

Considering a uniform temperature in the whole system, (2) can relate pressures and volumes.

$$
P_{i} v_{i}=C_{i}
$$

Where $v_{i}$ is the volume of gas in the $i^{\text {th }}$ HPA and $C_{i}$ is the gas constant, which can be considered equal for both sides if the HPAs have the same pressure $\left(C_{i}=C_{1}=C_{2}\right)$. In (3), the volumes are expressed as the sum of added oil volumes into the hydraulic circuit $\left(v_{a i}\right)$ and the oil coming from the cylinder $\left(S x_{p}\right)$, where $S$ represents the piston's area

$$
\begin{aligned}
& v_{1}=v_{1}(0)-v_{a 1}-S x_{p} \\
& v_{2}=v_{2}(0)-v_{a 2}+S x_{p}
\end{aligned}
$$

The instantaneous stiffness $K$ is the derivative of force generated by the difference of pressures in the cylinder with respect to displacement. Equation (4) shows that stiffness is a function of $v_{a i}$. If fluid is added to both chambers, the volumes of gas reduce and the pressures increase. Similarly, if fluid is subtracted from both chambers, the volume of gas increases and the pressure in both chambers reduces.

$$
K=\frac{\partial F_{p}}{\partial x_{p}}=C S^{2}\left(\frac{1}{v_{1}^{2}}+\frac{1}{v_{2}^{2}}\right)
$$

Figure 2 presents a schematic of the hydraulic circuit. This system is characterized by its simplicity, ease of assembly, low cost and its off-the-shelf elements. The main components are the hydraulic cylinder $(C y l l)$, a pair of HPAs of equal specifications(ACl-2), a hydraulic pump (HyS), two pressure gauges (PG1-2), four electro-proportional flow control valves

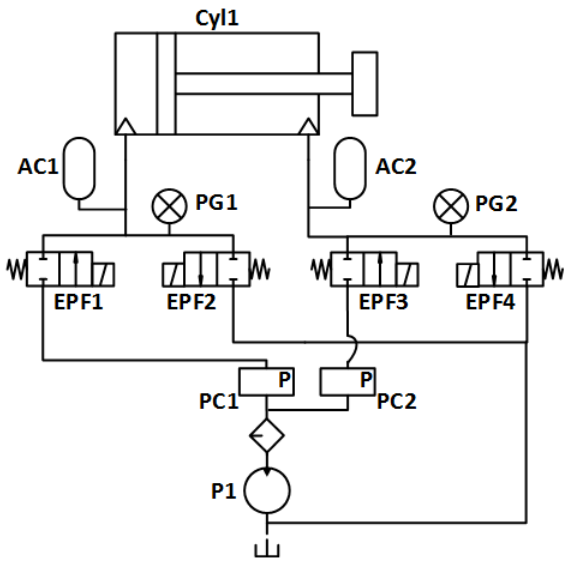

Fig. 2: Electro-hydraulic circuit

(EPF1-4) and two proportional pressure control valves (PCl2). A pair of flow control valves is used for each chamber in order to accurately drive the amount of oil in the system and to reduce manufacturing costs. The pressure control valves isolate the hydraulic input for each chamber of the cylinder.

\section{A. Model-based controller}

Several types of control laws have been implemented regarding the position and stiffness of BMVSA [10], [11]. These controllers work fine for a small working range (dual-coupled PID) or are computationally expensive (fuzzy control). An efficient model-based controller was developed in [15] and its briefly described next.

An encoder and a couple of pressure gauges provide feedback during the process. The time derivative of $v_{a i}$ describes the volumetric flow rate $\left(q_{i}\right)$. One constraint to be considered is that subtracted/added volume cannot be larger than the total volume of gas since the liquid fraction is considered incompressible. Equation (5) was deducted considering $u$ as spring's restoring force equal to $F_{p}$ and $x_{p}^{*}$ as the desired position, where $K_{E}$ is the characteristic gain of the spring and $u^{*}$ is the desired control signal. The error of position is stated in (6) and its derivative with respect to time in (7), where $\gamma$ is the tuning parameter to achieve minimal error.

$$
\begin{gathered}
u^{*}=-K_{E}\left(x_{p}-x_{p}^{*}\right) \\
e=u-u^{*} \\
\dot{e}=-\gamma e
\end{gathered}
$$

The error of pressure is used to control the stiffness, which is defined as the difference between the current pressures of the system and the ones related to desired stiffness $\left(P_{r e f}\right)$, as shown in (8) and (9), where $\lambda$ is the tuning parameter.

$$
e_{p}=\left(\frac{P_{1}+P_{2}}{2}-P_{r e f}\right)
$$




$$
\dot{e_{p}}=-\lambda e_{p}
$$

Expanding and solving (7) and $(9)$ for $q_{i}$ allows to control the position and stiffness of the system as shown in (10), (11), (12), (13), (14) and (15).

$$
\begin{gathered}
{\left[\begin{array}{l}
q_{1} \\
q_{2}
\end{array}\right]=\left[\begin{array}{c}
P_{1}{ }^{2} \\
-P_{2}{ }^{2}
\end{array}\right] D b_{x p}(t)+\left[\begin{array}{c}
P_{1}{ }^{2} \\
P_{2}{ }^{2}
\end{array}\right] D b_{p}(t)} \\
D=\frac{1}{{P_{1}}^{4}+P_{2}{ }^{4}} \\
b_{x p}(t)=\frac{C}{S}\left(-\gamma e+w_{x p}(t)\right) \\
b_{p}(t)=-2 C\left(\lambda e_{p}+w_{p}(t)\right) \\
w_{x p}(t)=-\frac{S^{2} \dot{x_{p}}}{C}\left(P_{1}{ }^{2}+P_{2}{ }^{2}\right)+\dot{u}^{*} \\
w_{p}(t)=\frac{S^{2} \dot{x_{p}}}{2 C}\left(P_{1}{ }^{2}+P_{2}{ }^{2}\right)-P_{r e f}
\end{gathered}
$$

Based on the previous equations, an array of hydraulic proportional flow valves are used to vary the pressures in both chambers.

\section{Modeling OF REVOlUte JOINT}

Thanks to the BMVSA's scalability, it is easy to use it instead of a hydraulic cylinder in order to gain variable stiffness. This allows the designer to try different mechanism architectures to develop more complex devices, like cabledriven joints. Based on the work done in [3], [6], [7], a simple antagonistic revolute joint was modeled, like the one displayed on Fig. 3 This tendon-driven mechanism is commonly actuated in three different modes:

1) One VSA pulls one cable and a passive device pulls the other one (spring, dead weight, etc.)

2) One stiff actuator is placed in series with a VSD for each cable

3) One VSA is used for each cable

The designed model uses the third mode to take advantage of the BMVSA stiffness range and maintain high-output force. The following assumptions were taken into account for developing the system: (i) the cables have uniform cross sections and are massless, (ii) there is not slippage between the cables and the pulley and (iii) BMVSAs can only pull. Pre-tensions $\left(T_{p_{r e}}\right)$ are set in the cables to avoid slackening during motion, these forces are equal and do not influence joint's stiffness. The tensions in each cable are defined as the addition of the tension created by the VSA and the pre-tension of the cable, as shown in (16).

$$
T_{i}=T_{V S A_{i}}+T_{\text {pre }_{i}}
$$

This approach has the following kinematic constraints due to the fixed cable length.

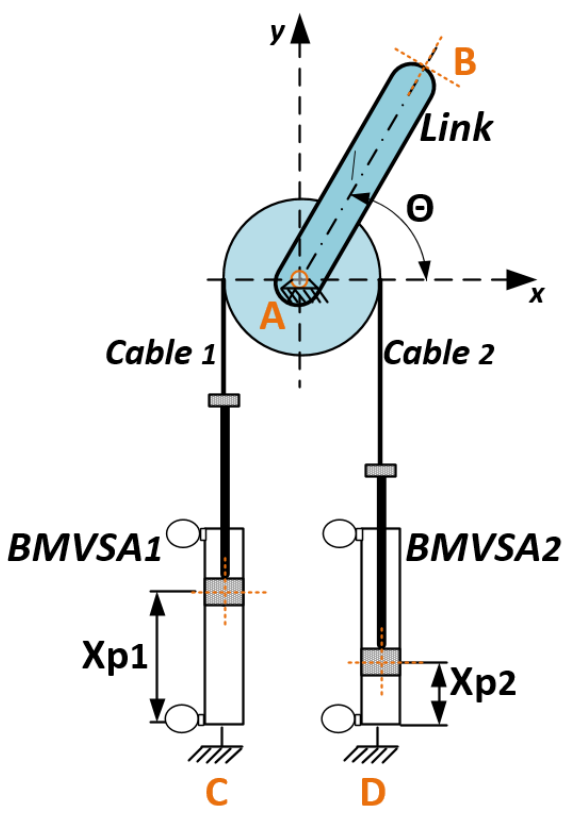

Fig. 3: Diagram of cable-driven revolute joint. The location of the components is important for the design and force distribution in the system: A-center of pulley; B- end on link; $\mathrm{C}$ and D- VSA fixation places

$$
\begin{gathered}
\delta l_{1}=r \theta-x_{p 1} \\
\delta l_{2}=-r \theta-x_{p 2}
\end{gathered}
$$

where $r$ is the radius of the pulley, $\theta$ is the rotation angle of the joint and $l_{i}$ the length of the ith cable. The output torque of the joint is defined as the difference of tensions in the cables as follows:

$$
\tau=r\left(T_{1}-T_{2}\right)
$$

Finally, the stiffness of the joint is expressed in the equation below.

$$
K_{\text {joint }}=r^{2}\left(K_{1}+K_{2}\right)
$$

\section{Simulation}

Only proprioceptive sensors of BMVSAs are taken into account for the simulation (encoders and pressure gauges); these sensors are enough to compute the joint angle $(\theta)$, its stiffness $\left(K_{\text {joint }}\right)$ and some external disturbance acting on the link (force or moment). The general control diagram is a leveled structure as shown in Fig. 4. The higher level contains the revolute joint controller (RJC), which receives the angle $\left(\theta_{d}\right)$ and joint stiffness $\left(K_{\text {joint }_{d}}\right)$ from the user and feeds the BMVSA controllers with desired positions $\left(x_{p_{i}}\right)$ and stiffness $\left(K_{i}\right)$. The actuator level controllers, developed in section II-A provide the desired flows signals to the BMVSA and receives information from the pressure sensors and the encoders; these measurements also contain information of external disturbances coming from the revolute joint as variations in the cable tensions $\left(T_{i}\right)$. To simplify the system, RJC calculates 


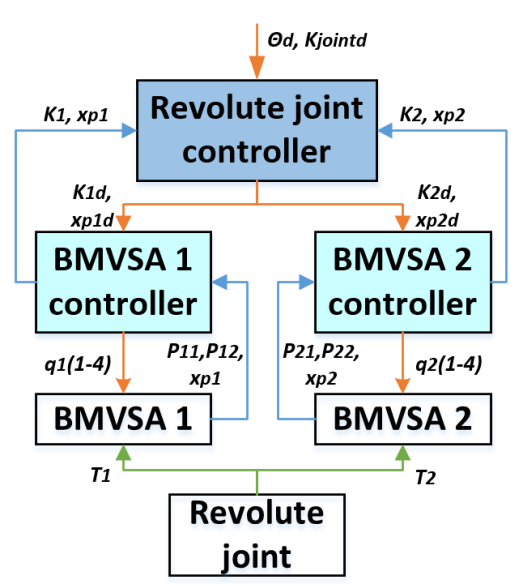

Fig. 4: Control block diagram. Green arrows represent physical interactions, orange connections transmit references and blue arrows stand for measured and control signals

$K_{i}$ equal for both actuators using eq. (19), and $x_{p_{i}}$ with eq. (17). For tension's distribution during the application of an external disturbance, it is done by checking the sign of the generated moment and pulling from the corresponding cable to compensate.

The following simulation was performed to study the stiffness and position of the joint using two equal BMVSAs with parameters given in Tab. I] where $v_{i}(0)$ and $v_{\text {imin }}$ are physical characteristics of real HPAs used in previous embodiments of the VSA. Figure 5 displays the reachable stiffness range of a single BMVSA, while Fig. 6 shows the conversion of the actuator stiffness into the cable-driven joint. It is noteworthy how the use of two BMVSA in the mechanism significantly increases the stiffness range.

The tests had an external disturbance represented by a moment with a square wave shape with $0.5 \mathrm{Nm}$ amplitude and baseline on $0 \mathrm{Nm}$ at $0.1 \mathrm{~Hz}$, as shown in Fig. 7. Position in the actuators is measured from left end of the cylinder to its right end. The angle is given as a sinusoidal signal with $\pi / 4$ radians amplitude and baseline on $\pi / 2$ radians at $0.1 \mathrm{~Hz}$, as displayed

TABLE I: Simulation parameters

\begin{tabular}{ll}
\hline \hline Parameter & Value \\
\hline$r$ & $0.0477 \mathrm{~m}$ \\
$l_{i}$ & $0.175 \mathrm{~m}$ \\
$l_{\text {link }_{\text {p }}}$ & $0.15 \mathrm{~m}$ \\
$T_{\text {pre }}$ & $1 \mathrm{~N}$ \\
$m$ & $1 \mathrm{Kg}$ \\
$K_{v}$ & $0.5 \mathrm{Ns} / \mathrm{m}$ \\
$K_{E}$ & $2500 \mathrm{~N} / \mathrm{m}$ \\
$S$ & $3.776 x 10^{-4} \mathrm{~m}^{2}$ \\
$C$ & $300 \mathrm{Pam}^{3}$ \\
$\gamma$ & -70 \\
$\lambda$ & -100 \\
$x_{p}(0)$ & $0.075 \mathrm{~m}$ \\
$x_{p_{\max }}$ & $0.15 \mathrm{~m}$ \\
$x_{p_{\min }}$ & $0 \mathrm{~m}$ \\
$v_{i}(0)$ & $1.5 x 10^{-4} \mathrm{~m}^{3}$ \\
$v_{i \min }$ & $v_{i}(0) / 4 \mathrm{~m}^{3}$ \\
\hline
\end{tabular}

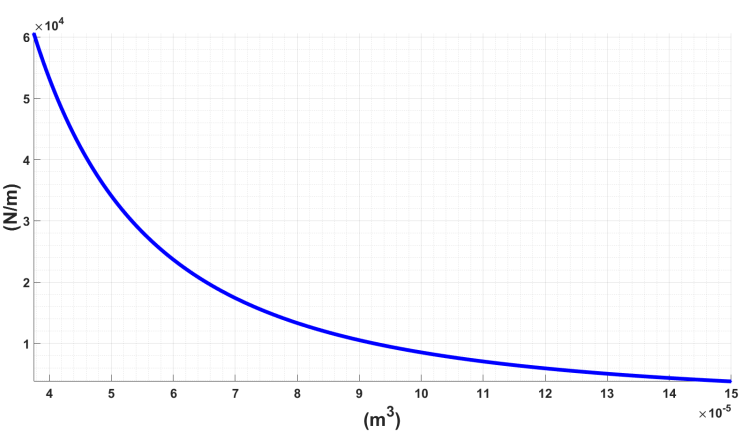

Fig. 5: BMVSA stiffness range with respect to compressed volume of gas

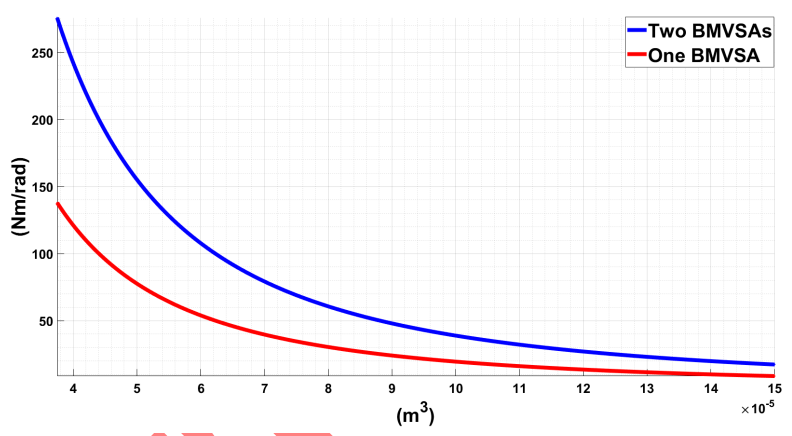

Fig. 6: Cable-driven joint stiffness range using one or two BMVSA with respect to compressed volume of gas

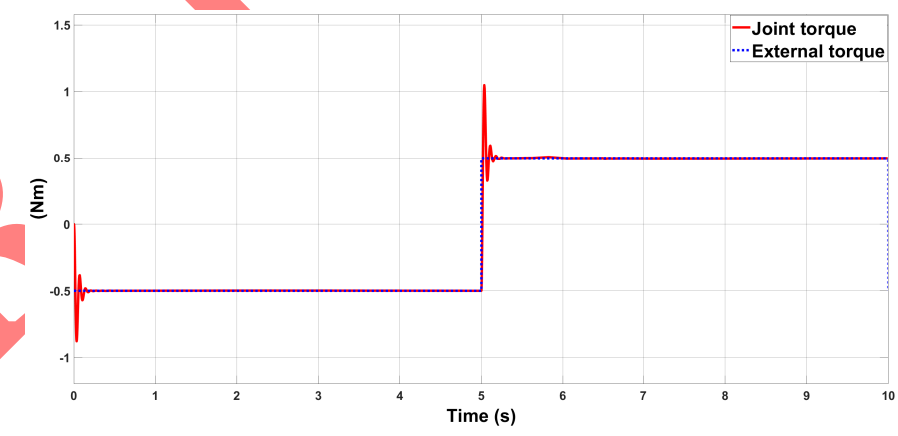

Fig. 7: Joint torque. The joint torque is able to compensate the external torque with a minimum settling time

in Fig. 8, while the desired stiffness is a sinusoidal signal with $25 \mathrm{Nm} /$ radians amplitude and baseline on $50 \mathrm{Nm} /$ radians at 0.1 $\mathrm{Hz}$, as depicted in Fig. 9 .

The outcome of the simulations proves that RJC provided correct references to actuator controllers (Figs. 11 and 12) allowing the system to track position and stiffness even if the desired signals to follow are nonlinear. Figure 10 displays the position of point $\mathrm{B}$ in X-Y plane; the importance of this point is due to its potential use as origin for a subsequent joint in a serial chain. Figures 7 and 13 reflect how the system is able to compensate an external disturbance by logically changing the tensions in the cables. It is worth to note that the controller prioritized stiffness tracking over position tracking, rendering the system unable to reach the position reference 


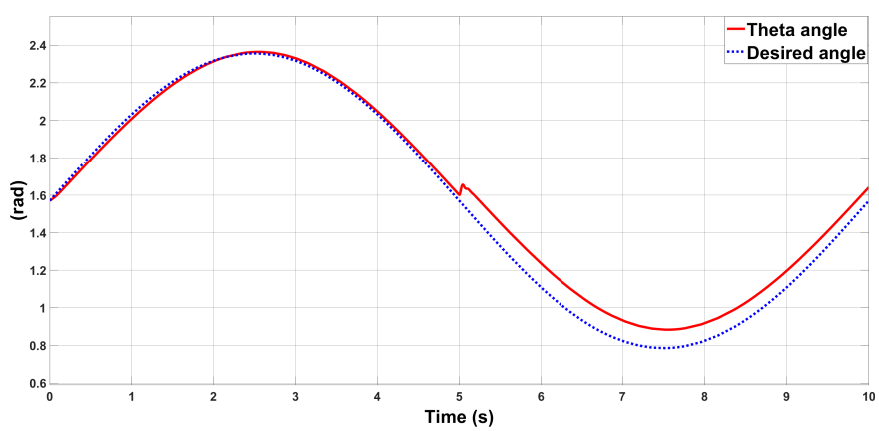

Fig. 8: Joint angle. The desired angle is properly tracked but not reached due to the low stiffness of the system

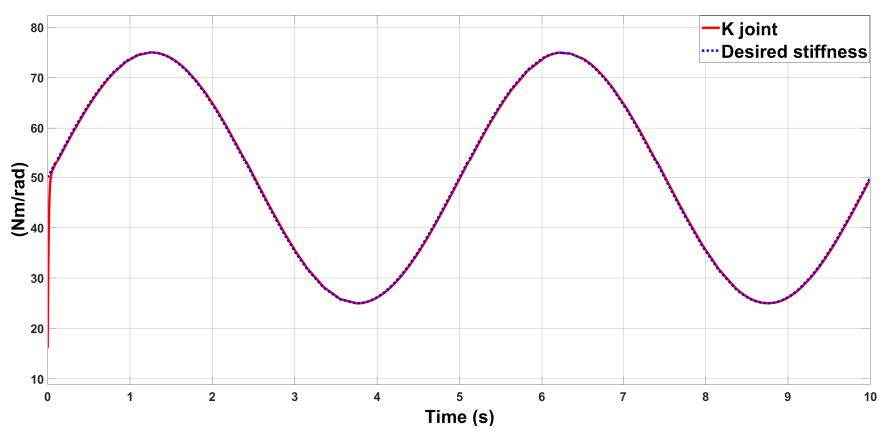

Fig. 9: Joint stiffness tracking

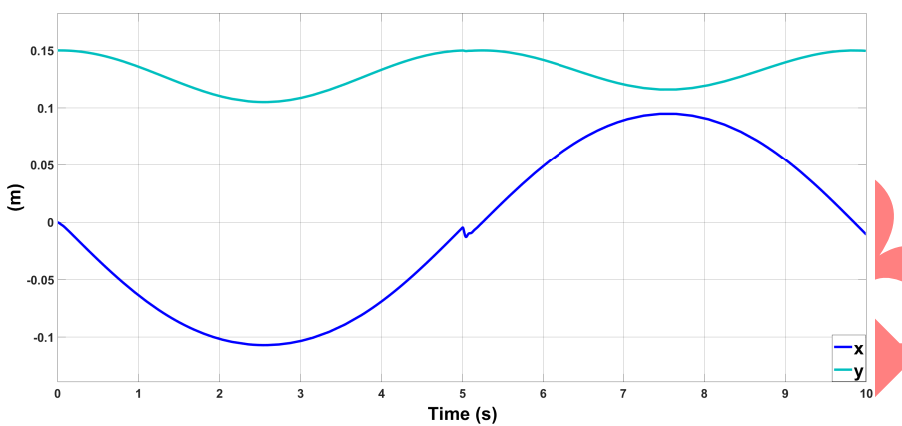

Fig. 10: Position of point B

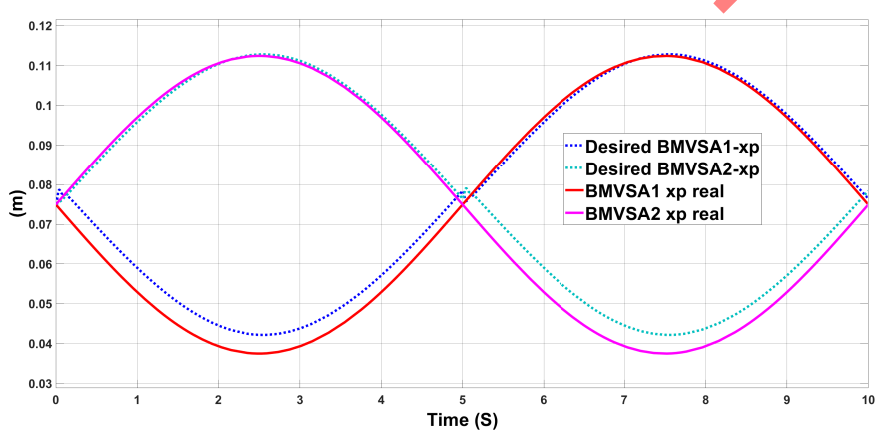

Fig. 11: BMVSAs position tracking

in the presence of disturbances, while the desired mechanism stiffness is always obtained, see Figs. 8, 11, 9 and 12 .

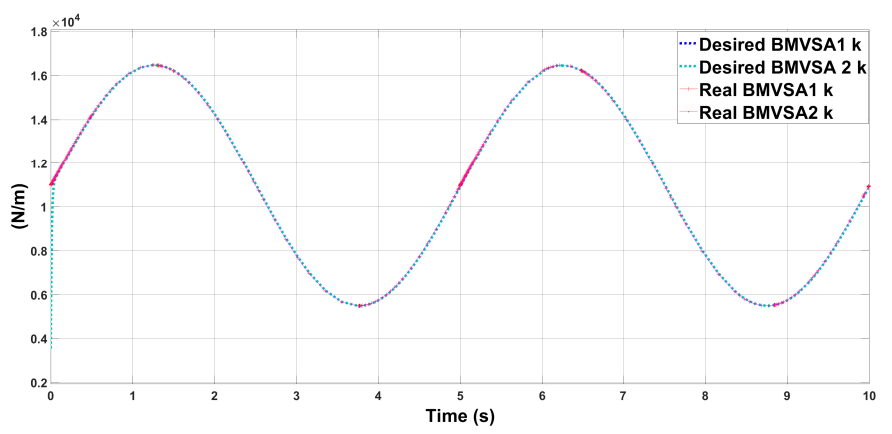

Fig. 12: BMVSAs stiffness tracking

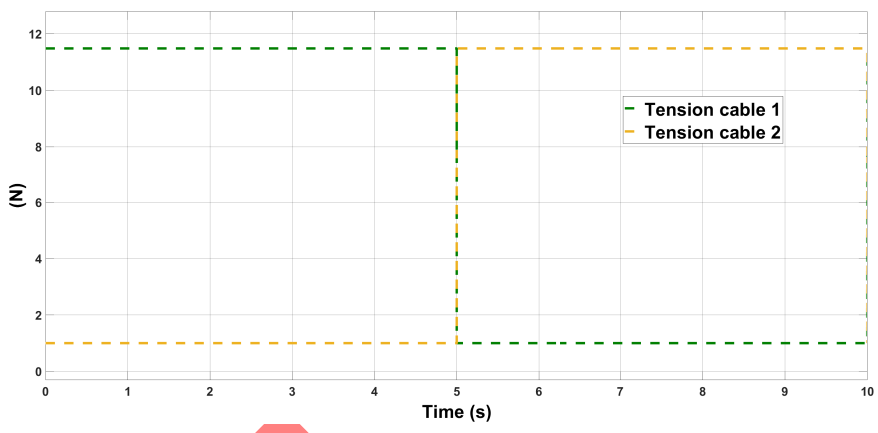

Fig. 13: Tensions in cables. The tensions are never zero due to pre-tensioning

\section{Conclusions}

Joint stiffness depends on the computed references for the BMVSAs. The hydraulic nature of BMVSA provides the possibility to have a high output force, while the nonlinearity of the gas provides a wider range of stiffness than the VSAs using traditional elastic components. The cable-based revolute mechanism was modeled to take full advantage of the BMVSA characteristics to create a light weight and high output power joint. The results obtained from simulations demonstrate that the proposed controller approach can accurately track desired stiffness and approximate the desired position, even when an external force acts on it. Further work needs to be developed regarding the influence of the output link's mass, friction and load's inertia.

A more accurate model including stiffness of the cable's material and the masses of the cables, pulley and link is being developed. This new approximation is expected to provide better understanding of the existing forces and help with the selection of components for prototype manufacturing.

\section{REFERENCES}

[1] B. Vanderborght, A. Albu-Schaffer, A. Bicchi, E. Burdet, D. G. Caldwell, R. Carloni, M. Catalano, O. Eiberger,W. Friedl, G. Ganesh, et al. "Variable impedance actuators: A review," Robotics and autonomous systems, vol. 61 (12), pp. 1601-1614, 2013.

[2] R. Van Ham, T. G. Sugar, B. Vanderborght, K. W. Hollander, and D. Lefeber, "Compliant actuator designs", IEEE Robotics and Automation Magazine, vol. 16 (3), 2009.

[3] Y. Wang, G. Yang, K. Yang, C. Zhang and T. Zheng, ”Design optimization for a 2-DOF cable-driven joint with large stiffness range," 2017 12th IEEE Conference on Industrial Electronics and Applications (ICIEA), Siem Reap, 2017, pp. 264-269. 
[4] W. B. Lim, S. H. Yeo, G. Yang and I. -. Chen, "Design and analysis of a cable-driven manipulator with variable stiffness," 2013 IEEE International Conference on Robotics and Automation, Karlsruhe, 2013, pp. 45194524. doi: 10.1109/ICRA.2013.6631219

[5] E. A. Brackbill, Y. Mao, S. K. Agrawal, M. Annapragada and V. N. Dubey, "Dynamics and control of a 4-dof wearable cabledriven upper arm exoskeleton," 2009 IEEE International Conference on Robotics and Automation, Kobe, 2009, pp. 2300-2305. doi: 10.1109/ROBOT.2009.5152545

[6] Zhou X, Jun S, Krovi V. A Cable Based Active Variable Stiffness Module With Decoupled Tension. ASME. J. Mechanisms Robotics. 2015;7(1):011005-011005-5. doi:10.1115/1.4029308.

[7] Jeong, H., Kang, B., Cheong, J. Stiffness analysis and experimental validation of modular-type hybrid antagonistic tendon-driven joint systems. Robotica, 36(11), 1680-1700. (2018). doi:10.1017/S0263574718000632

[8] M. Zoppi, "Method for adapting stiffness in a variable stiffness actuator," US Patent 8991 169, March 31, 2015.

[9] B. Han, M. Zoppi, and R. Molfino, "Variable impedance actuation using biphasic media," Mechanism and Machine Theory, vol. 62, pp. 1-12, 2013.

[10] B. Han, M. Zoppi, and R. Molfino, "Variable impedance actuation mesomicro physical models and rotation link using biphasic media," Procedia Engineering, vol. 64, pp. 1020-1029, 2013.

[11] B. Han, "Variable impedance actuation using biphasic media," Ph.D Thesis dissertation, University of Genoa, 2013.

[12] L. Le, M. Zoppi, M. Jilich, R. Camoriano, D. Zlatanov, and R. Molfino, "Development and analysis of a new specialized gripper mechanism for garment handling," in ASME 2013 International Design Engineering Technical Conferences and Computers and Information in Engineering Conference, pp. V06BT07A013-V05BT08A023, 2013.

[13] L. Le, M. Zoppi, M. Jilich, B. Han, D. Zlatanov, and R. Molfino, "Application of a biphasic actuator in the design of a robot gripper for garment handling," in ASME 2014 International Design Engineering Technical Conferences and Computers and Information in Engineering Conference, pp. V05BT08A022-V05BT08A022, 2014.

[14] L. Le, M. Zoppi, M. Jilich, B. Han, D. Zlatanov, and R. Molfino, "Application of a biphasic actuator in the design of the clopema robot gripper," Journal of Mechanisms and Robotics, vol. 7, pp. 011011(1-8), 2015.

[15] J.H. Lugo, G. Cannata, M. Zoppi, and R. Molfino, "Modeling and Simulation of a Biphasic Media Variable Stiffness Actuator," International Journal of Mechanical Engineering and Robotics Research, 2018.

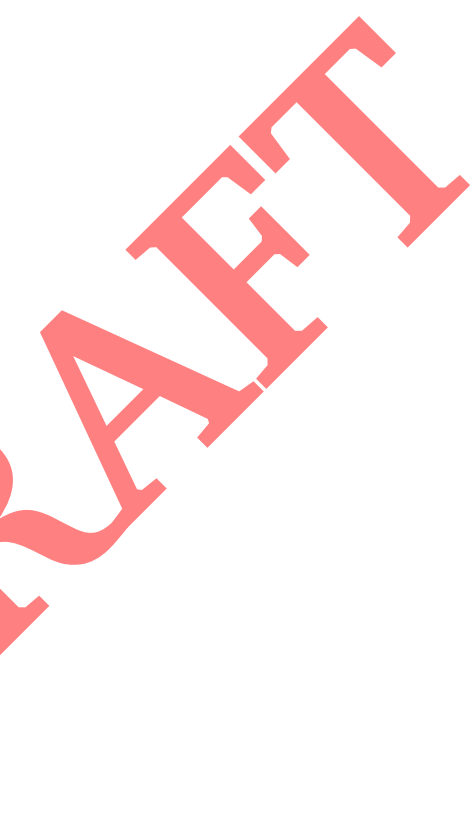

